\title{
EchoGéo
}

$31 \mid 2015$

Glocal Ethiopia

\section{Perspectives locales et globales pour la gestion des terres des régions montagneuses du Nord de l'Éthiopie}

\author{
Jan Nyssen, Amaury Frankl, Jean Poesen, Amanuel Zenebe et Jozef
} Deckers

\section{(2) OpenEdition}

Journals

Édition électronique

URL : https://journals.openedition.org/echogeo/14171

DOI : 10.4000/echogeo.14171

ISSN : 1963-1197

Éditeur

Pôle de recherche pour l'organisation et la diffusion de l'information géographique (CNRS UMR 8586)

Référence électronique

Jan Nyssen, Amaury Frankl, Jean Poesen, Amanuel Zenebe et Jozef Deckers, «Perspectives locales et globales pour la gestion des terres des régions montagneuses du Nord de l'Éthiopie », EchoGéo [En ligne], 31 | 2015, mis en ligne le 10 avril 2015, consulté le 31 juillet 2021. URL : http:// journals.openedition.org/echogeo/14171 ; DOI : https://doi.org/10.4000/echogeo.14171

Ce document a été généré automatiquement le 31 juillet 2021.

EchoGéo est mis à disposition selon les termes de la licence Creative Commons Attribution - Pas d'Utilisation Commerciale - Pas de Modification 4.0 International (CC BY-NC-ND) 


\title{
Perspectives locales et globales pour la gestion des terres des régions montagneuses du Nord de l'Éthiopie
}

\author{
Jan Nyssen, Amaury Frankl, Jean Poesen, Amanuel Zenebe et Jozef \\ Deckers
}

Les auteurs remercient les agriculteurs éthiopiens, les universités et administrations pour leur intérêt et pour leur coopération à la recherche, tout comme les collègues suivants pour les discussions fructueuses et contributions scientifiques (ordre alphabétique) : Alemtsehay Tsegay, Bart Muys, Biadgilgn Demissie, Dereje Teklemariam, Elise Monsieurs, Ellen Meire, Enyew Adgo, Etefa Guyassa, Gebeyehu Taye, Gebreyohannes Girmay, Hanne Govaers, Hanne Hendrickx, Hans Bauer, Henok Kassa, Ine Vandecasteele, Jan Moeyersons, Johan Van de Wauw, Jozef Naudts, Karen De Geyndt, Karen Vancampenhout, Kassa Amare, Kassa Teka, Katrien Descheemaeker, Kindeya Gebrehiwot, Kristine Martens, Kristine Walraevens, Liën Romeyns, Ludwin Poppe, Lys Moulaert, Mathias Vanmaercke, Mekete Dessie, Miro Jacob, Mitiku Haile, Neil Munro, Nigussie Haregeweyn, Paolo Billi, Raf Aerts, Romha Assefa, Sil Lanckriet, Silke Broidioi, Stefaan Dondeyne, Stéphanie de Mûelenaere, Tesfaalem Gebreyohannes, Tesfamichael Gebreyohannes, Tesfay Araya, Wim Clymans et Wim Cornelis. VLIR-UOS, FWO Flandres-Belgique, CIUF et le Fonds de Recherche Spécial de l'Université de Gand (Belgique) et NUFFIC (Pays-Bas) ont mis des ressources financières à la disposition des chercheurs.

\section{Introduction}

1 Depuis au moins trois millénaires, les paysages tourmentés du Nord de l'Éthiopie (illustration 1) ont été dégradés par l'agriculture. Comme les sols ne sont pas limoneux, et portent souvent une couverture pierreuse dense (Virgo et Munro, 1978 ; Nyssen et al., 2002), les profils du sol n'ont pas encore été entièrement tronqués par l'érosion généralement associée aux labours agricoles. Les pratiques agricoles sont bien adaptées à l'environnement : l'outil de labourage, l'araire ou mashrasha, a été développé pendant la période de haute technologie Axoumite; les systèmes de culture s'adaptent 
harmonieusement aux chaînes (catena) de sols (Nyssen et al., 2008a), et les systèmes agricoles sont bien adaptés aux variations interannuelles des conditions pluviométriques (Frankl et al., 2013 ; Pietsch et Machado, 2014). Alors que, d'un point de vue technique et dans les conditions traditionnelles, l'adaptation agricole à la variabilité des sols et du climat est pratiquement optimale, la gestion des terres a depuis longtemps été entravée par l'inégalité d'accès aux terres (Lanckriet et al., 2015) et par un pâturage le plus souvent libre, y compris la vaine pâture. Dans cette synthèse, nous aborderons le passé et le futur de la gestion des terres dans le Nord de l'Éthiopie. Comment l'état des sols et des forêts a-t-il changé au cours du siècle dernier ? Quelles stratégies de gestion des terres pourraient être adoptées afin d'améliorer la production durable des ressources des sols, des eaux et des forêts ? Les agriculteurs éthiopiens ont démontré leurs qualités de bons gestionnaires individuels des terres, mais sont-ils à même d'être de bons planificateurs de l'utilisation des terres? Quelle incidence cela at-il sur les moyens de subsistance de la population? Les perspectives globales (changement climatique, mondialisation) seront également prises en compte.

Illustration 1- . Carte orohydrographique de l'Éthiopie du Nord avec indication des lieux cités dans le texte

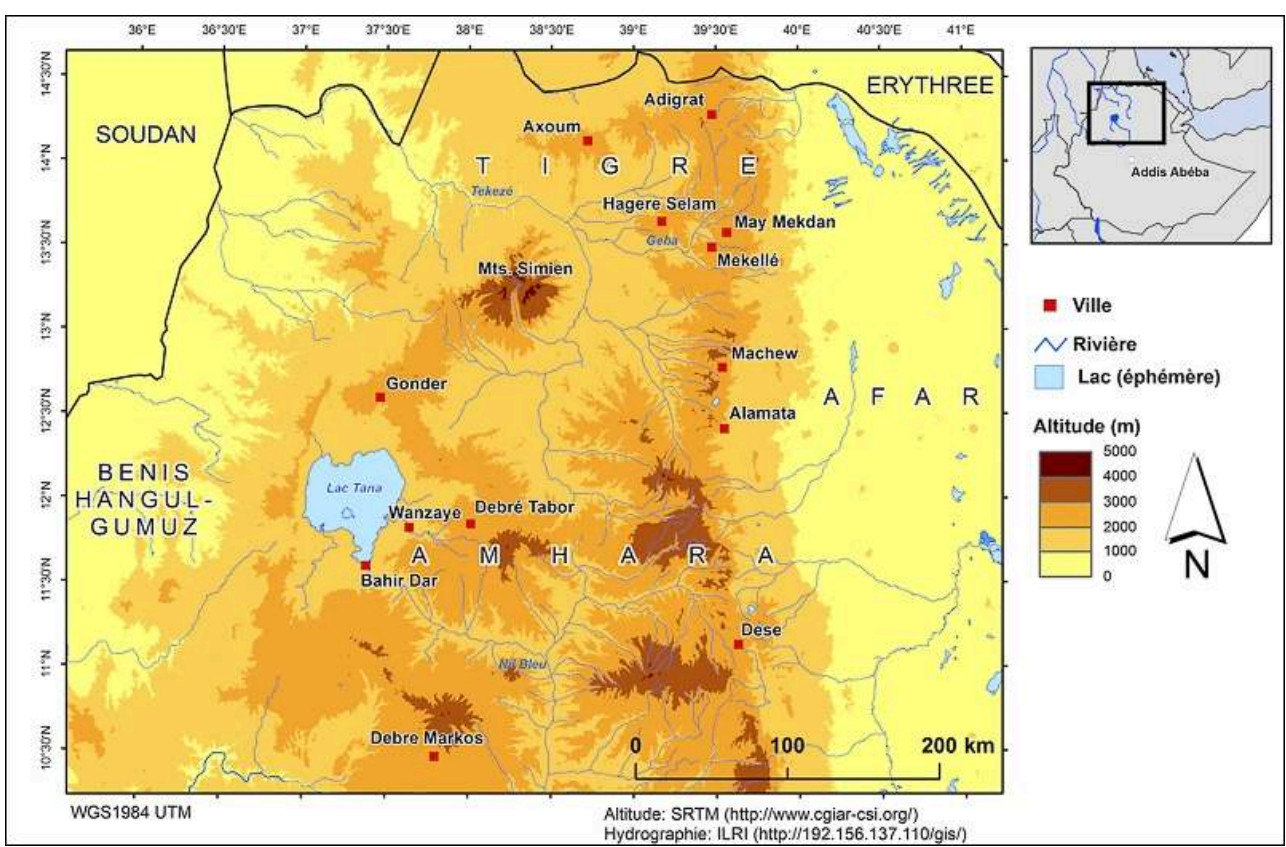

\section{Méthodologies de recherche}

$2 \mathrm{Au}$ cours des trois dernières décennies, toute une gamme de méthodologies de recherche environnementale a été déployée dans les régions montagneuses du Nord de l'Éthiopie afin d'acquérir une meilleure compréhension des transformations du paysage, en mettant l'accent sur les processus, leurs vitesses et leur variabilité spatiale. Des prises de vue multi-spectrales par satellites, y compris des estimations de pluviométrie déterminées par la NOAA (Frankl et al., 2013), ont également été utilisées. Récemment, l'interprétation de plus de 350 photographies historiques de paysages éthiopiens, ainsi que de clichés représentant les mêmes paysages à l'heure actuelle ont permis de cartographier l'utilisation et le couvert des terres depuis la fin du XIX ${ }^{e}$ siècle, 
et de développer des évolutions chronologiques décrivant le couvert végétal et la conservation des sols et de l'eau (Nyssen et al., 2014). La récupération des photographies aériennes italiennes de l'Ethiopie, réalisées en 1935-41, la reconstruction de la photogrammétrie utilisée à cette époque, et la fusion de ces résultats avec les images obtenues par télédétection, afin d'obtenir une analyse géomorphologique rétrospective (Frankl et al., 2015) ont constitué les dernières étapes de recherche.

\section{La dégradation historique des terres en Éthiopie}

Des études chronologiques menées dans le sud de l'Éthiopie, à l'origine densément couvert de forêts (Gobeze et al., 2009), mais où actuellement la déforestation se poursuit encore (Wood, 1993), montrent une très importante augmentation des pertes de sol au cours des dernières décennies. Certains résultats d'études paléo-environnementales indiquent que, dans les montagnes du Nord, le défrichement et la régénération de la forêt constituent un processus cyclique qui se produit depuis 3000 ans (illustration 2; Eshetu et Högberg, 2000 ; Darbyshire et al., 2003 ; Lamb et al., 2007). Ces conclusions basées sur des études palynologiques sont en contradiction avec un paradigme encore largement accepté qui veut qu'« en 1900 (ou 1930, ou 1965, ...), $40 \%$ (ou $45 \%$, ou $30 \%, \ldots$ ) de la superficie de l'Éthiopie (ou de l'Éthiopie du Nord, ou du Tigré, ou de l'Érythrée, ...) était sous forêt. Suite à la démographie galopante (ou l'agriculture archaïque, ou l'ignorance des populations, ...), il ne reste que $1 \%$ (ou $0,5 \%$, ou $3 \%, \ldots$ ) de ce couvert forestier ». Différents auteurs ont cherché la source de telles affirmations sans jamais en trouver la trace (Pankhurst, 1995 ; Wøien, 1995 ; Gascon, 1998 ; McCann, 1998 ; Ritler, 2003 ; Nyssen et al., 2004 ; Boerma, 2006 pour l'Érythrée). Au contraire, déjà en 1946, Logan estimait qu'au maximum $5 \%$ des hauts plateaux éthiopiens étaient boisés. Prenant en compte le potentiel climatique et édaphique du couvert forestier en Éthiopie, $40 \%$ correspondrait approximativement au couvert forestier au moment de l'introduction de l'agriculture (Von Breitenbach, 1961). 
Illustration 2 - Forêt de Juniperus âgée de 100 à 200 ans, à Kuskuam, près de Debre Tabor

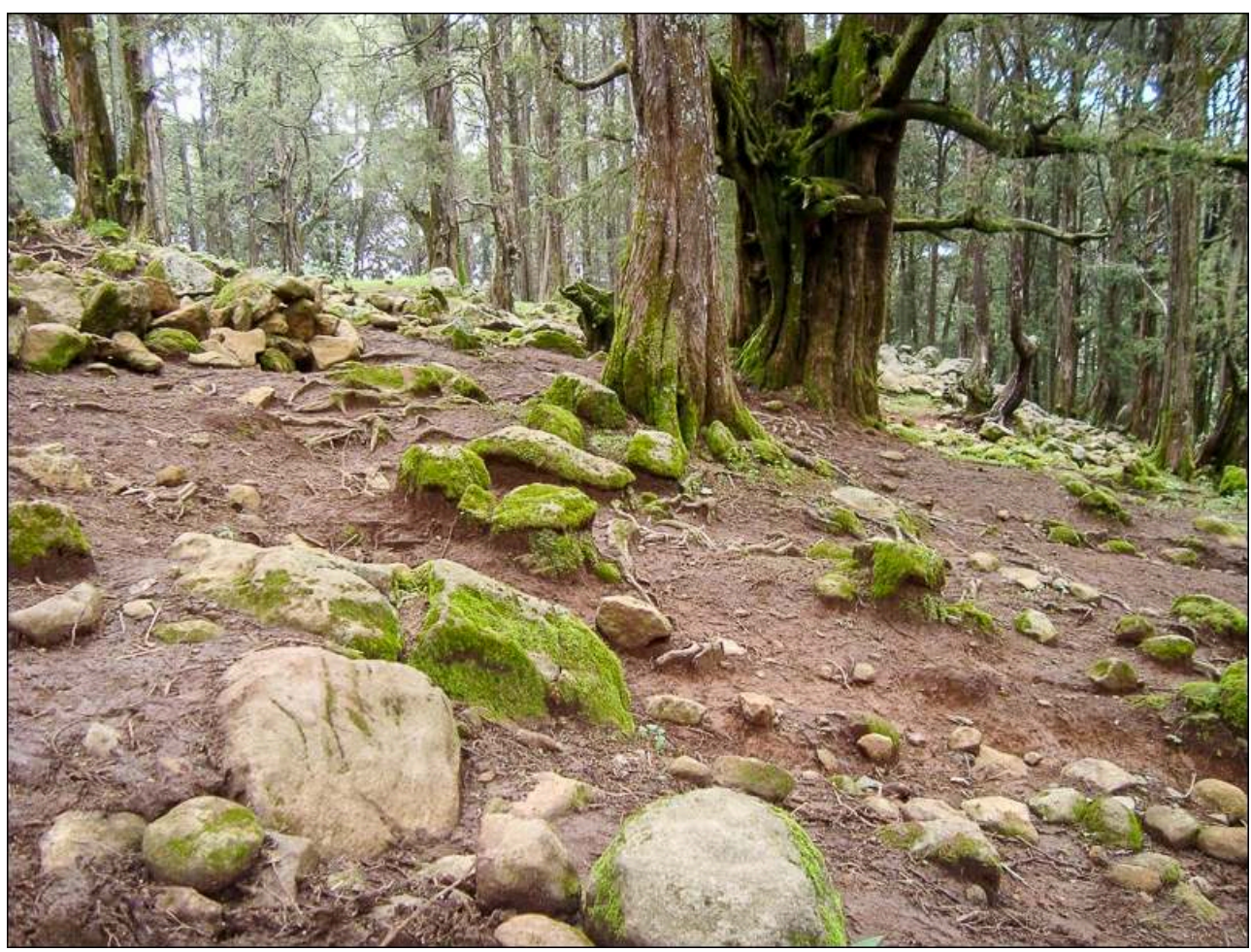

Des marques de charrue sur de gros blocs rocheux, qui sont souvent en position de piédestal, indiquent que cette forêt de Juniperus âgée de 100 à 200 ans, à Kuskuam, près de Debre Tabor, s'est développée sur une terre agricole fortement érodée. La repousse forestière est également mise en évidence par l'arbre plus ancien et bien ramifié au centre de la photographie, qui autrefois poussait dans une zone ouverte.

Auteur : J. Nyssen, juillet 2011.

4 Depuis les premières études réalisées par Hurni $(1975,1978,1983)$ et Virgo et Munro (1978), de nombreuses études sur l'érosion en Éthiopie ont été menées. Les études sur des parcelles expérimentales par Herweg et Ludi (1999), SCRP (2000), Nyssen et al. (2009c) et Gebeyehu Taye et al. (2013) montrent des taux d'érosion très variables (0-200 t/ha/an) en lien avec la topographie et l'affectation des sols. Dans les zones caractérisées par une faible densité de structures de conservation du sol et de l'eau (SWC), les taux de pertes de sol sont de l'ordre de $10 \mathrm{t} / \mathrm{ha} / \mathrm{an}$ à l'échelle du bassin versant et ont pour origine essentiellement (pour les 2/3) l'érosion en nappe et en rigoles (Nyssen et al., 2009a) (illustration 3). Concernant les bassins versants, Herweg et Stillhardt (1999), SCRP (2000), Nigussie Haregeweyn et al., (2008), Nyssen et al. (2009a), ou encore Vanmaercke et al. (2010) ont établi que le coefficient d'écoulement comme le transport de sédiments (exprimé en $\mathrm{t} / \mathrm{km} / \mathrm{an}$ ) diminue avec la taille grandissante des bassins. Ceci est à relier au fait que dans des bassins plus larges, il existe de plus vastes plaines alluviales dans lesquelles beaucoup de sédiments sont déposés.

5 Les ravines de ruissellement étudiées par Nyssen et al. (2006); Daba et al. (2003) et Frankl et al. (2012) ont connu une expansion rapide. Des reconstructions environnementales nous montrent que depuis la fin du XIX siècle, des ravines d'érosion étaient présentes, bien qu'elles apparaissent stabilisées sur des photos datant de 1935 ; une phase accentuée de ravinement a ensuite recommencé dans les années 1960 (Frankl et al., 2011). Des études de mouvements de masse par Moeyersons et al. (2008), et Van Den Eeckhaut et al. (2009) indiquent qu'il s'agit généralement de 
réactivations de glissements de terrain plus anciens, qui étaient bien plus larges et qui sont supposés dater d'une période présentant moins d'écoulement superficiel, comme avant l'introduction de l'agriculture il y a environ 3000 ans.

Illustration 3 - Erosion en rigoles (voir flèches) dans un champ à Wanzaye non loin du Lac Tana, au milieu de la saison de pluies de 2013

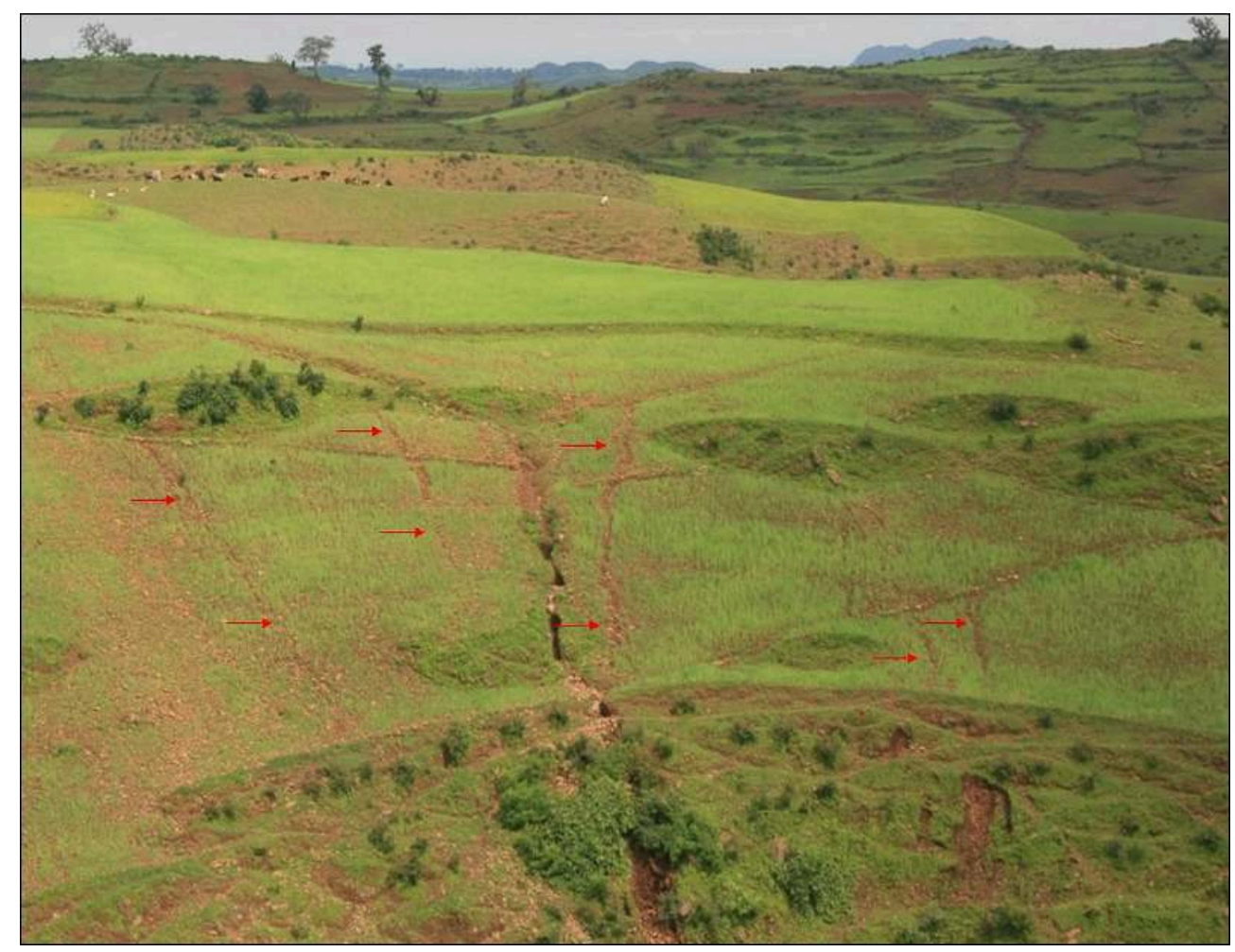

L'exploitant tente d'empêcher cette forme d'érosion en guidant l'eau de ruissellement vers un ravin, qui subira une expansion à son tour.

Auteur : Elise Monsieurs.

6 En fonction du climat et de la topographie du lieu, la profondeur de la nappe phréatique saisonnière et les conditions hydrologiques, et par conséquent le ruissellement et la réponse en termes d'érosion du sol, peuvent varier (Tebebu et al., 2010 ; Monsieurs et al., 2015).

\section{Conservation physique et biologique du sol}

7 Les vitesses de ravinement et la densité de drainage décroissent depuis environ les années 2000, du fait de la croissance des actions de conservation du sol et de l'eau, et de l'amélioration du couvert végétal (Frankl et al., 2011). La fertilité du sol reste un enjeu, d'autant que les apports organiques sont systématiquement éliminés des terres agricoles du fait de la vaine pâture et de l'utilisation du fumier comme carburant (Kraaijvanger et Veldkamp, 2015). L'un des inconvénients des structures de conservation physique est par ailleurs leur contribution à la prolifération de certaines espèces de rongeurs (Nyssen et al., 2007 ; Meheretu Yonas et al., 2014).

8 En ce qui concerne le couvert forestier, même si le mythe d'un couvert forestier de $40 \%$ vers 1900 a été démenti depuis dix ans (voir ci-dessus), la photographie répétitive a 
montré que vers la fin du XIX siècle, les paysages étaient déjà au moins aussi arides qu'aujourd'hui (Nyssen et al., 2009b). Depuis 1975, le couvert arboré s'est cependant amélioré sur $90 \%$ des paysages analysés (Nyssen et al., 2008b ; de Mûelenaere et al., 2014). Des terres anciennement communales ont été mises en défens (Aerts et al., 2009), à des fins de restauration de la forêt et de conservation des terres. La mise en place de ces « exclos» a été rendue possible par l'introduction d'une importante réforme de la propriété foncière au cours des années 1980. Il en résulte que d'importantes terres agricoles féodales situées aux fonds des vallées et dans d'autres zones planes ont été partagées avec les agriculteurs locaux, réduisant ainsi le besoin ressenti par les paysans pauvres d'établir des terres agricoles marginales sur les flancs des collines. En ces lieux, les « exclos » ont alors pu être établis après la réforme agraire (Dessalegn Rahmato, 1994 ; Lanckriet et al., 2015). Bien qu'elle ait été imposée par le pouvoir central, l'entrée en vigueur des mises en défens se fait plutôt de manière ascendante. La localisation, les surfaces, les règlements municipaux relatifs aux restrictions et à la gestion, ainsi que l'installation et le paiement de gardiens, sont généralement décidés au niveau de la communauté locale (Bedru Babulo et al., 2012). Globalement, les villageois participent de manière convaincante à la reforestation et à d'autres activités de conservation (Kumasi et Asenso-Okyere, 2011). La diffusion de l'eucalyptus reste un goulot d'étranglement pour la biodiversité : les bénéfices de la plantation de ces arbres se retrouvent essentiellement au niveau des agriculteurs individuels, alors que les effets environnementaux néfastes de cet arbre très consommateur d'eau sont supportés par les communautés (Selamyihun Kidanu et al., 2005; Zenebe Mekonnen et al., 2007; Tilashwork Chanie et al., 2013).

9 Les preuves de changements environnementaux, mises en évidence par 361 photographies jumelées, montrent que les conditions de la végétation boisée, du sol et des structures de conservation de l'eau et de la gestion de la terre, font apparaître une situation plus dégradée dès la fin du XIX siècle qu'actuellement (illustrations 4a et $4 b)$. Le couvert formé par les arbres indigènes est une exception notable : il a atteint un pic au cours des années 1930 (illustrations 5 a et 5b), a ensuite diminué, puis a atteint un deuxième pic au début du XXI ${ }^{\mathrm{e}}$ siècle (Nyssen et al., 2014). 
Illustrations 4a et 4b - Vues au pied de l'Amba Fekada au nord d'Adigrat en 1868 et en 2008
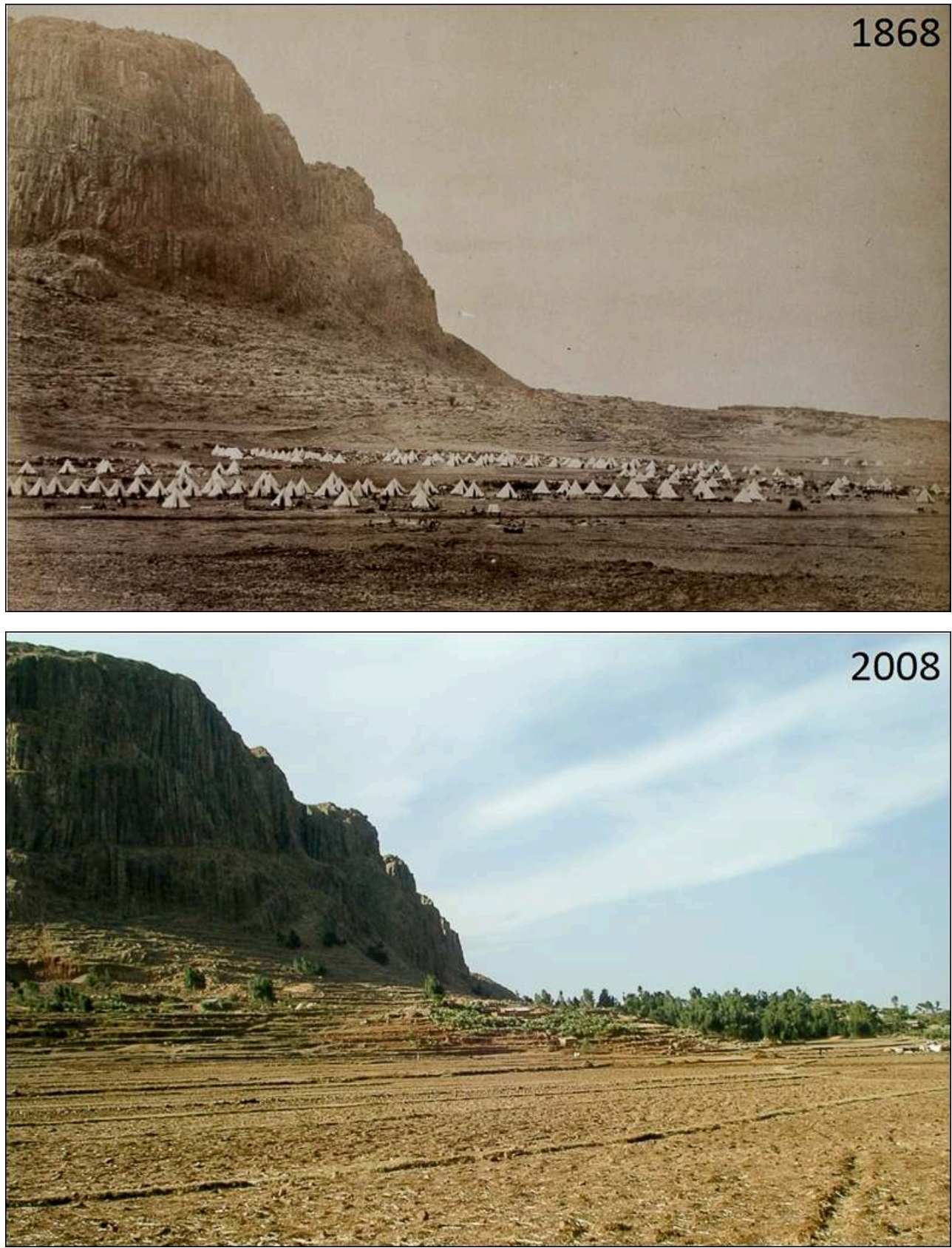

Lorsque l'armée britannique, en campagne contre l'empereur Tewodros, établissait son camp au pied de l'Amba Fekada au nord d'Adigrat en 1868, il n'y avait pas d'arbres dans le paysage et le village de Fekada était déjà présent sur la basse colline à droite sur la photo. Depuis, le village s'est fortement étendu et des eucalyptus ont été plantés autour des maisons. Sur le piedmont de gauche des terrasses agricoles ont été établies. Nyssen et al. (2009b) présentent le contexte de ces photos historiques et une analyse semi-quantitative.

Auteurs : Photographie historique (c) Kings Own Museum à Lancaster, Angleterre ; photographie de 2008 par J. Nyssen. 

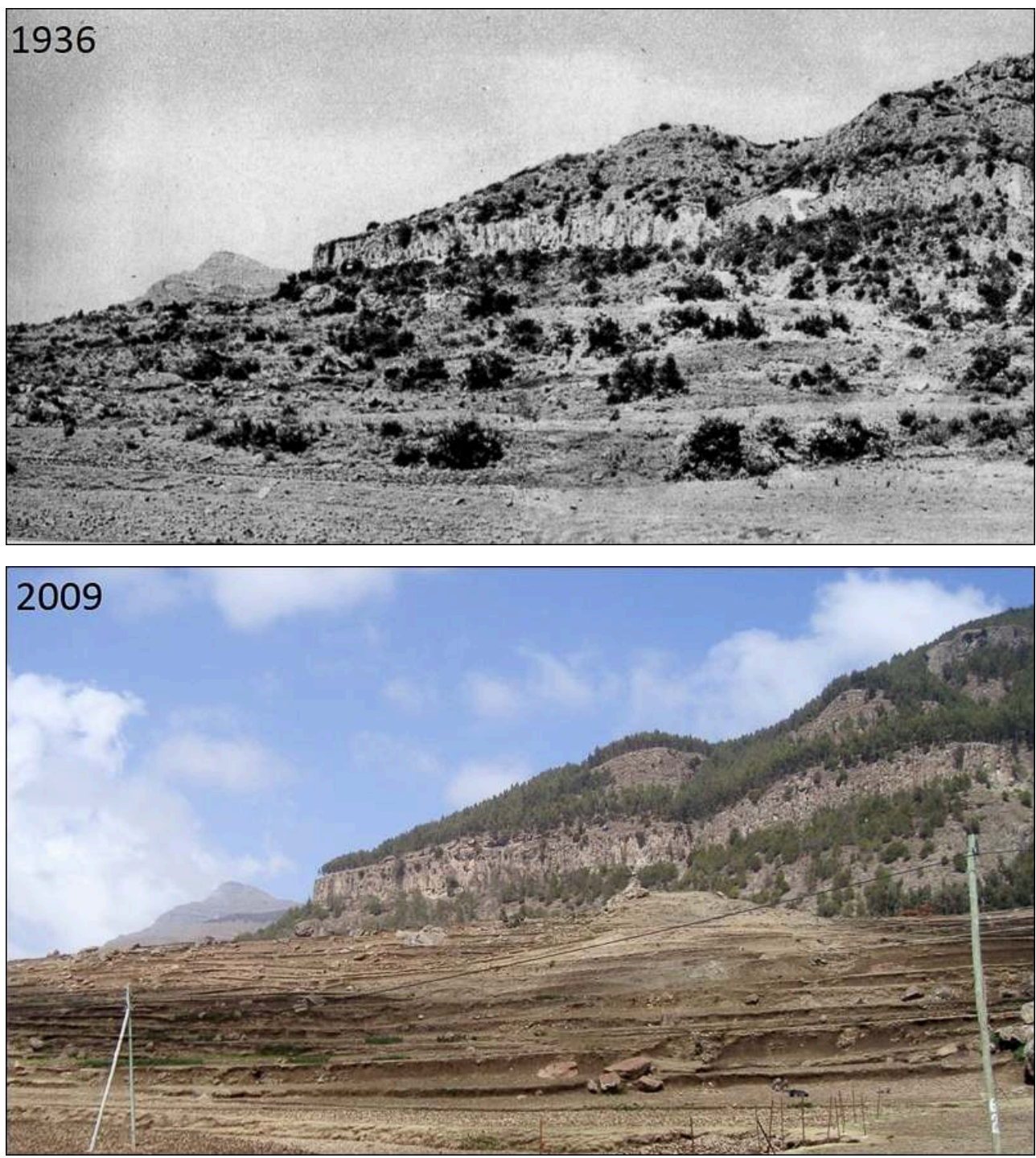

D'importants changements en affectation du sol et en couvert végétal ont eu lieu autour de la falaise basaltique de Debar près de Maychew. En 1936, des genévriers (Juniperus procera) éparpillés et rabougris étaient les témoins de la déforestation qui avait eu lieu. Dans la période après 1936, tous les arbres restants sur les terrains ondulants de l'avant-plan ont dû faire place à des terrains agricoles où des rideaux indiquent les limites parcellaires. Le couvert forestier a fort peu changé sur les pentes au pied de la falaise ; par contre les sommets ont été reboisés dans les années 1980 avec une forêt mixte Juniperus-Eucalyptus.

Auteurs : Photographie historique Merla et Minucci, 1936 ; photographie de 2009 par J. Nyssen.

\section{Les efforts de conservation et leurs effets sur la disponibilité de l'eau}

10 La récupération de l'eau in situ et la construction de petits réservoirs ont fortement contribué à réduire les apports en sédiments (Nigussie Haregeweyn et al., 2008, 2013) et les coefficients de ruissellement (CR) à l'échelle du bassin versant, à faire croître les niveaux des nappes phréatiques (Nyssen et al., 2010; Gebremedhin Berhane et al., 2013), et à améliorer les conditions de vie, comme cela a également été signalé ailleurs en 
Afrique (Wildemeersch et al., 2015). Or, d'importantes différences de CR n'ont pas pu être mises en évidence entre les sous-bassins versants du bassin de Geba de $5000 \mathrm{~km}^{2}$, en raison vraisemblablement de la mise en place d'activités de conservation des sols et des eaux (CSE) dans tous les bassins (Amanuel Zenebe et al., 2013). Dans les cas de grandes reconversions forestières en particulier, telles que celle de la zone située en amont d'Alamata, ces effets apparaissent très nettement, surtout en termes de réduction des inondations en aval et des évolutions de la morphologie des chenaux fluviaux (i.e. profondeur et largeur) (Tesfaalem Gebreyohannes et al., 2015). Des expériences d'agriculture de conservation en exploitation se sont révélées prometteuses, notamment en termes de diminution de la réponse en pertes de sols et en ruissellement (McHugh et al., 2007; Tesfay Araya et al., 2012). Il s'agit maintenant de trouver les moyens les plus adaptés pour intensifier l'agriculture de conservation à l'échelle de régions plus étendues. Cela nécessiterait de mener des travaux de recherche sur le partage des connaissances et la prise en charge entre agriculteurs. Hormis ces activités de conservation, l'Éthiopie a fortement investi dans les intrants agricoles, notamment en ce qui concerne les engrais et des semences de meilleure qualité. En conséquence, la production alimentaire totale est maintenant plus élevée qu'elle ne l'a jamais été. De plus, entre 2005 et 2010 la production céréalière par habitant était de 60 \% supérieure à celle réalisée entre 1985 et 1990 (illustration 6).

Illustration 6 - Intensification agricole en Éthiopie

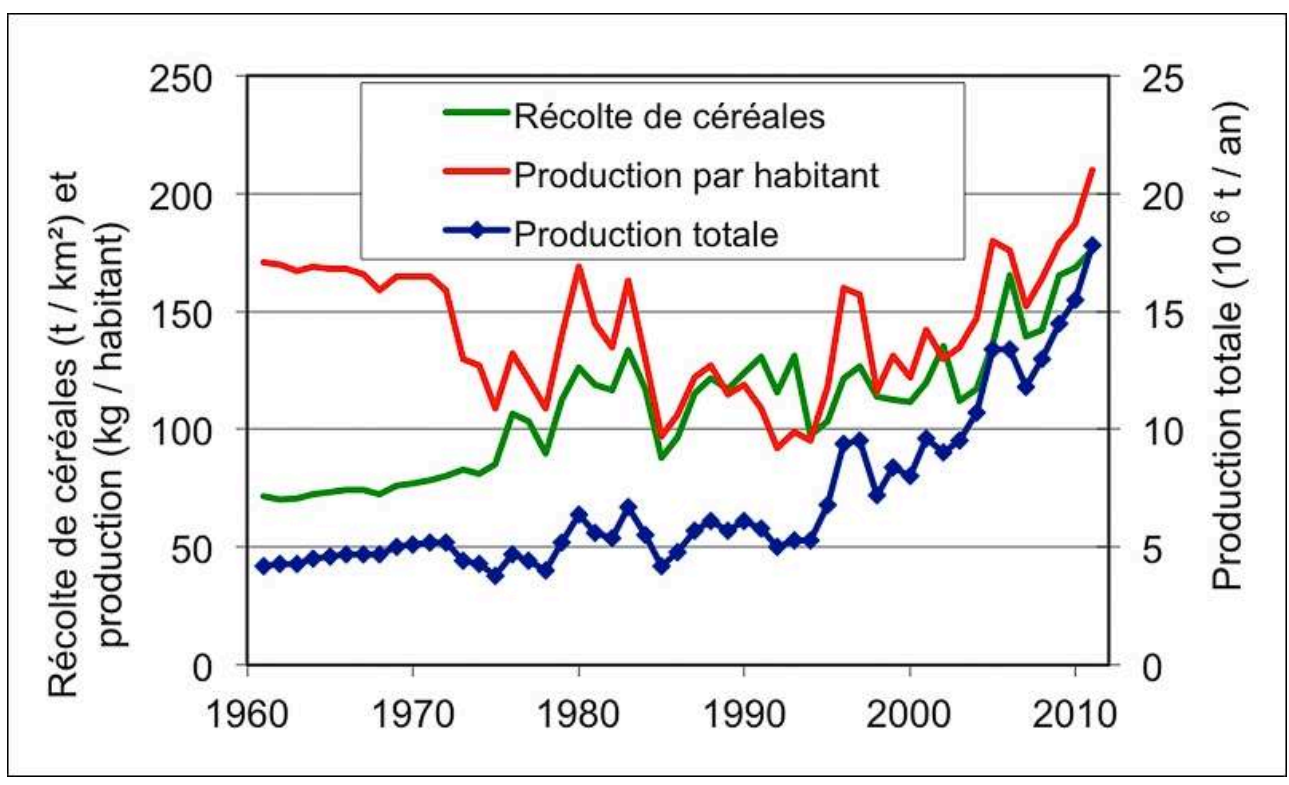

L'intensification agricole en Ethiopie est mise en évidence par les évolutions de la production céréalière (données recueillis depuis http://faostat.fao.org).

\section{Mondialisation économique et gestion des terres}

11 L'agriculture éthiopienne est partiellement intégrée au marché mondial. Le café est le principal produit d'exportation, et jusqu'au milieu des années 1970 la jouissance des terres, fréquemment basée sur le métayage, a permis l'accumulation et l'exportation de grands volumes de céréales (Holmberg, 1977; Koehn, 1979). Les tendances actuelles sont celles d'une forte croissance de l'exportation des fleurs et légumes, ainsi qu'une 
exportation en plein essor du khat (Al-Hebshi et Skaug, 2005), un narcotique léger dont la culture est très consommatrice d'eau (Almas et Scholz, 2006). L'acquisition des terres à grande échelle, en particulier en dehors des hauts plateaux (illustration 7), est actuellement le fait principalement de sociétés indiennes, chinoises, allemandes, et de la diaspora éthiopienne établie dans des pays riches (Dereje Teklemariam et al., 2015). Ce processus est-il semblable à celui de l'accaparement des terres qui se produisait jusqu'en 1975 sur les hauts plateaux, les deux tiers des terres étant alors entre les mains de l'Église et des propriétaires féodaux ? Malgré l'engagement du gouvernement à fournir des terres agricoles étendues, des études récentes indiquent que sur les 11,5 millions d'hectares de terres, à peine 2,5 millions ont été transférés à des investisseurs internationaux (MoA, 2012). Des observations in situ, par exemple dans la région de Benishangul-Gumuz, indiquent un faible niveau d'investissement dans ces terres qui sont encore souvent occupées par la forêt et utilisées par des communautés locales. Dans la plupart des projets, l'étude d'impact sur l'environnement, obligatoire, n'a pas été effectuée. Les investisseurs sont en concurrence avec les communautés locales non seulement pour les terres, mais aussi pour l'eau, et les communautés locales perçoivent peu d'avantages en termes d'emplois, de sécurité alimentaire ou d'infrastructure (Lavers, 2012 ; Dereje Teklemariam et al., 2015).

Illustration 7 - Ferme à capitaux étrangers, établie récemment près de Gublak dans le BenishangulGumuz

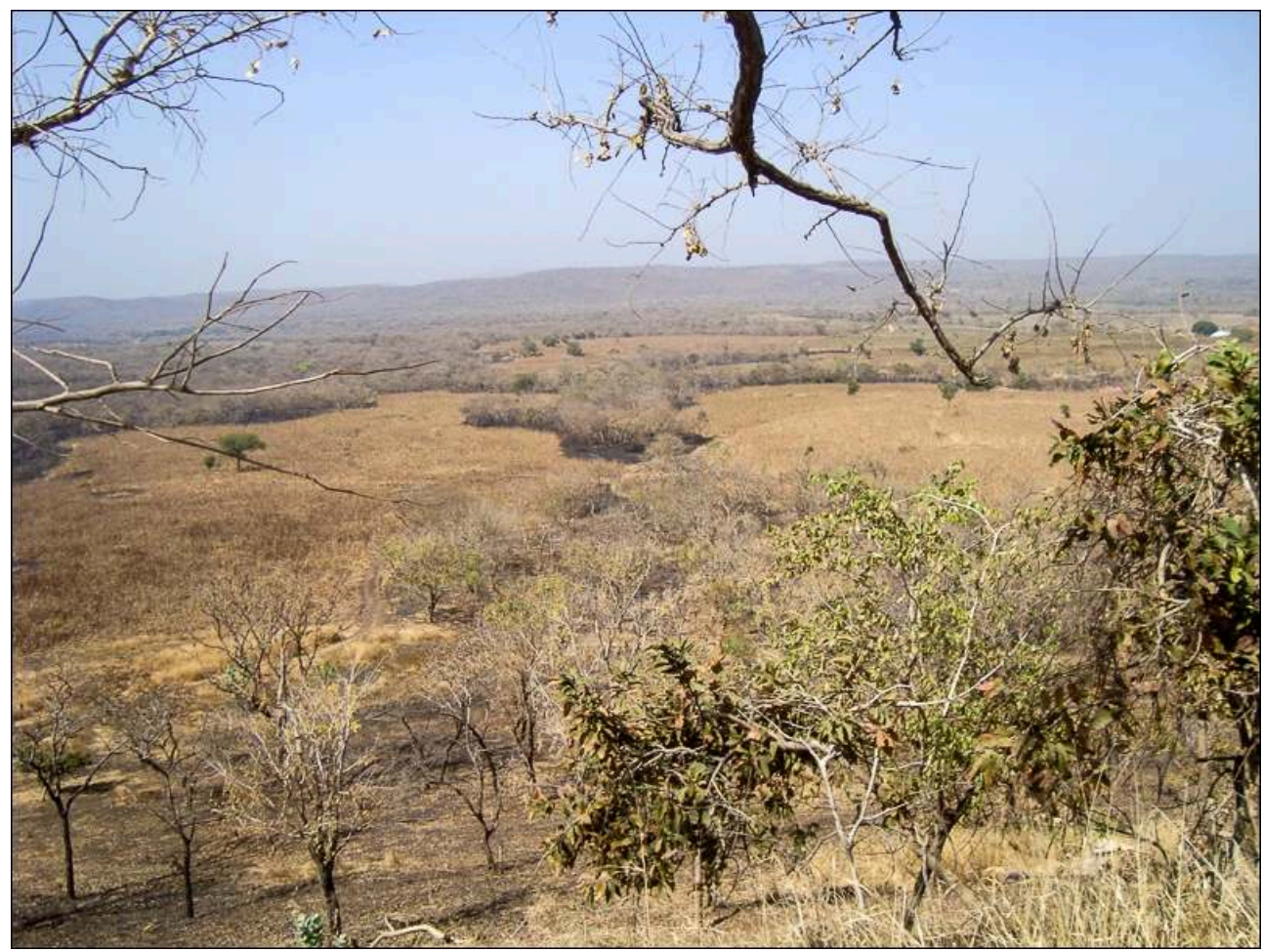

La savane arborée, traditionnellement soumise au brûlis annuel, a été remplacée par des champs de coton. Ici, l'exploitant a bien respecté une des clauses du contrat qui est de laisser $50 \mathrm{~m}$ de végétation naturelle le long du thalweg. Les travailleurs de la ferme viennent cependant de brûler ces bandes forestières dans le but de pouvoir récolter le miel des abeilles sauvages.

Auteur : J. Nyssen, janvier 2015. 


\section{Changement climatique mondial}

Le chapitre concernant l'Afrique du récent rapport du GIEC (Niang et al., 2014) précise que la température de l'air a augmenté sur tout le continent africain durant le $\mathrm{XX}^{\mathrm{e}}$ siècle et qu'elle augmentera d'au moins $2^{\circ} \mathrm{C}$ vers la fin du siècle (Funk et al., 2012; Conway et Schipper, 2011). Les changements anticipés en précipitations sur le continent sont variables, mais les hauts plateaux éthiopiens sont explicitement mentionnés dans le rapport du GIEC comme une région pour laquelle les projections indiquent une augmentation probable des précipitations et des précipitations extrêmes vers la fin du XXI e siècle (de Wit et Stankiewicz, 2006 ; Moise et Hudson, 2008 ; Shongwe et al., 2011 ; Lanckriet et al., 2012). Des observations ponctuelles sur les flancs du Ferrah Amba $\left(12.86^{\circ} \mathrm{N}, 39.489^{\circ} \mathrm{E}\right)$ indiquent que ce phénomène conduit déjà à un déplacement en amont de la frontière agro-climatique pour le blé. L'impact du réchauffement climatique sur la limite forestière supérieure devra être distingué de l'influence de l'occupation humaine près des zones afro-alpines (Jacob et al., 2015). Le rapport du GIEC insiste beaucoup sur les conséquences des changements anticipés pour les systèmes de production agricoles en Éthiopie et cite à plusieurs reprises en exemple les programmes d'adaptation mis en place dans ce pays (Zerihun Berhane et Prowse, 2013 ; Heltberg et al., 2009; Belay Simane et al., 2012). Une augmentation importante de la réponse en ruissellement (et par conséquent la densité de drainage et de l'érosion des sols) est attendue dans les zones d'openfield du Nord de l'Éthiopie (de Wit et Stankiewicz, 2006 ; Lanckriet et al., 2012). L'hydrologie sera fortement affectée dans le bassin du Nil (Tazebe Beyene et al., 2010 ; McCartney et Michael Menker, 2012). En outre, le volume plus important des eaux de ruissellement pourrait être plus favorable aux cultures irriguées sur les terres basses, notamment dans les grabens marginaux de la vallée du Rift.

Illustration 8 - Changements environnementaux

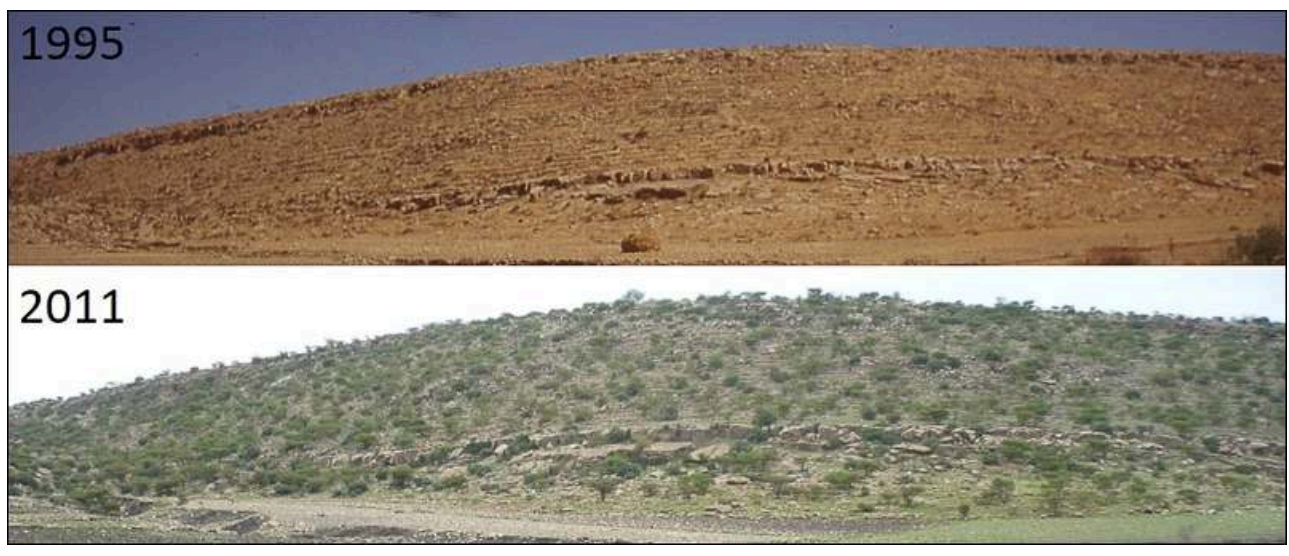

Entre 1995 et 2011 la végétation secondaire a fortement repoussé sur ce terrain calcaire près de May Makden. II s'agit ici d'un « enclos » saisonnier où la coupe du bois est interdite, mais la pâture autorisée pour les bœufs dans les mois précédant les labours. De tels changements environnementaux sont, au Tigré, plutôt la règle que l'exception.

Auteurs : photographie 1995, F. Dramis; photographie 2011, A. Frankl. 


\section{Conclusions et perspectives}

13 L'Éthiopie du Nord est bien visible sur la carte mondiale lorsqu'il s'agit des recherches portant sur les ressources environnementales et la mise en œuvre d'une gestion durable des terres (GDT - Mitiku Haile et al., 2006). Les piliers de la GDT se basent sur la bonne connaissance que les agriculteurs ont de leur environnement biophysique, et incluent le développement forestier dans les zones critiques (Descheemaeker et al., 2006, 2009) (illustration 8) sur des surfaces suffisamment grandes, comme cela a été mis en évidence par des modifications spectaculaires de l'utilisation des terres qui se sont produites sur l'escarpement de la vallée du Rift. Les activités de conservation des sols et de l'eau augmentent également les infiltrations lors d'orages brefs mais intenses, et améliorent la situation en ce qui concerne les inondations, l'érosion des sols et les eaux souterraines. Le régime foncier actuel, qui cherche à établir une meilleure équité au niveau de la propriété foncière, privilégie la solidarité entre agriculteurs pour ce qui concerne les activités de gestion des bassins versants communaux (Kumasi et AsensoOkyere, 2011). Toutes ces mesures font partie d'une approche globale qui a permis de fortement augmenter les récoltes.

D'un point de vue conceptuel, il est important d'avoir une bonne compréhension du bilan hydrologique. Par ailleurs, pour les processus relatifs aux nutriments, aux sédiments et aux eaux, il est important de comprendre quelles en sont les sources et les puits à diverses échelles spatiales. Ces principes sont à la base d'une mise en œuvre réussie des activités de gestion des bassins versants au Nord de l'Éthiopie. À cet égard, l'impact de la gestion du bassin versant de May Zeg-zeg à Hagere Selam a pu être suivi de près (Nyssen et al., 2009a; Walraevens et al., 2009; Nyssen et al., 2010) (illustration 9) et des scénarios de développement futur ont pu être élaborés (Lanckriet et al., 2012), comprenant notamment le développement de mesures de conservation in situ, telles que l'agriculture de conservation. 


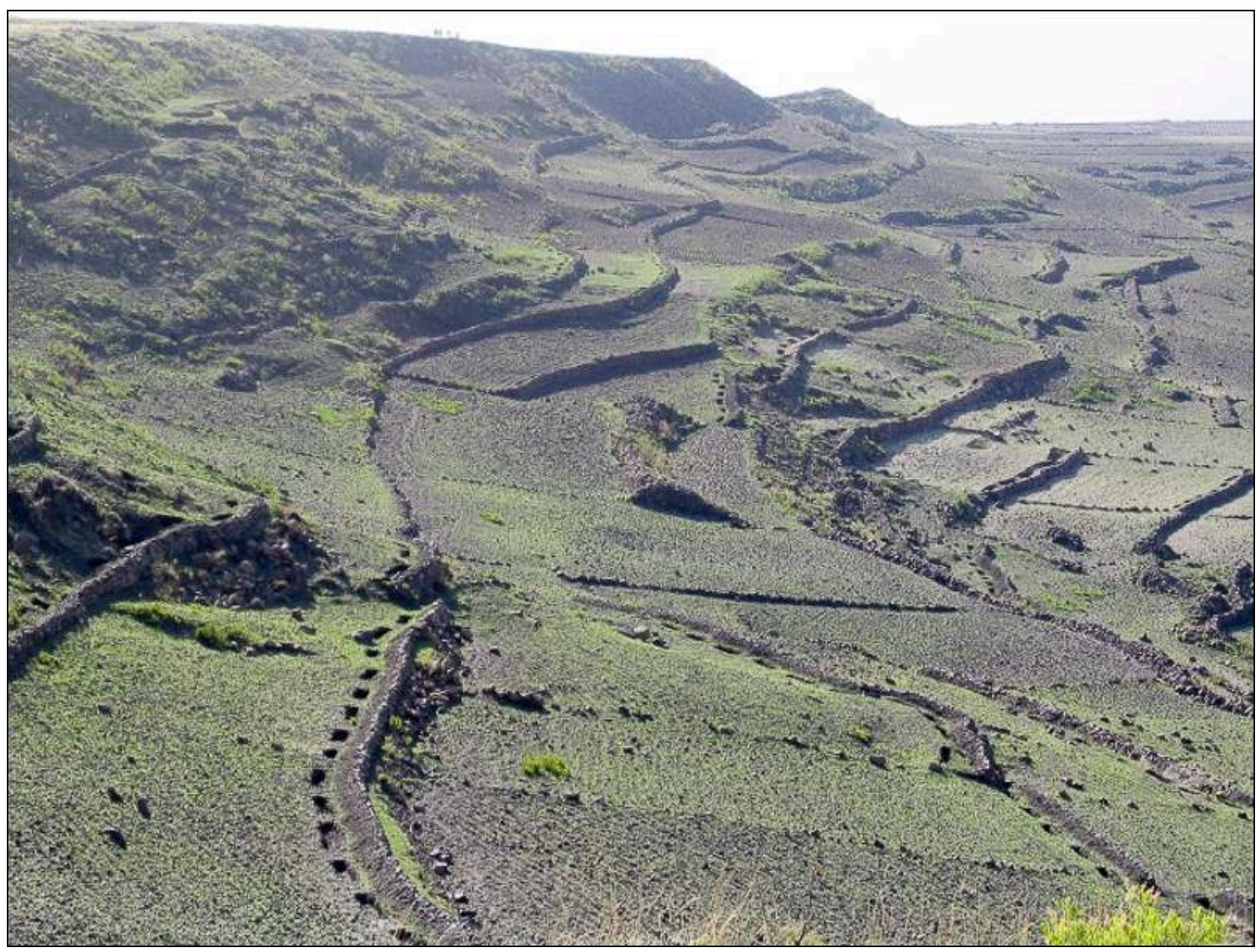

Les tranchées cloisonnées présentes à l'arrière des murets de pierre augmentent les infiltrations et diminuent le ruissellement dans le bassin versant.

Auteur: K. Herweg, mai 2005.

\section{BIBLIOGRAPHIE}

Aerts R., Nyssen J., Mitiku Haile, 2009. On the difference between "exclosures" and "enclosures" in ecology and the environment. Journal of Arid Environments, 73, p. 762-763.

Al-Hebshi N., Skaug N., 2005. Khat (Catha edulis) -an updated review. Addiction Biology, 10, p. 299-307.

Almas AAM., Scholz M., 2006. Agriculture and water resources crisis in Yemen: need for sustainable agriculture. Journal of Sustainable Agriculture, 28, p. 55-75.

Amanuel Zenebe, Vanmaercke M., Poesen J., Verstraeten G., Nigussie Haregeweyn, Mitiku Haile, Kassa Amare, Deckers J., Nyssen J., 2013. Spatial and temporal variability of river flows in the degraded semi-arid tropical mountains of northern Ethiopia. Zeitschrift für Geomorphologie, 57, p. 143-169.

Bedru Babulo, Muys B., Nigussie Haregeweyn, Descheemaeker K., Deckers J., Poesen J., Nyssen J., Mathijs E., 2012. Cost-benefit analysis of soil and water conservation measure: The case of exclosures in northern Ethiopia. Forest Policy And Economics, 15, p. 27-36. http://dx.doi.org/ 10.1016/j.forpol.2011.09.008 
Belay Simane, Zaitchik B.F., Desalegn Mesfin., 2012. Building climate resilience in the Blue Nile/ Abay Highlands: A framework for action. International Journal of Environmental Research and Public Health, 9, p. 610-631.

Boerma P., 2006. Assessing forest cover change in Eritrea-a historical perspective. Mountain Research \& Development, 26, p. 41-47.

Conway D., Schipper ELF., 2011. Adaptation to climate change in Africa: Challenges and opportunities identified from Ethiopia. Global Environmental Change, 21, p. 227-237.

Daba S., Rieger W., Strauss P., 2003. Assessment of gully erosion in eastern Ethiopia using photogrammetric techniques. Catena, 50, p. 273-291.

Darbyshire I., Lamb H., Umer M., 2003. Forest clearance and regrowth in northern Ethiopia during the last 3000 years. The Holocene, 13, p. 537-546.

De Wit M., Stankiewicz J., 2006. Changes in surface water supply across Africa with predicted climate change. Science, 311, p. 1917 - 1921.

de Mûelenaere S., Frankl A., Mitiku Haile, Poesen J., Deckers J., Munro R.N., Veraverbeke S., Nyssen J., 2014. Historical landscape photographs for calibration of Landsat land use/cover (1972) in the northern Ethiopian highlands. Land Degradation \& Development, 25, p. 319-335.

Dereje Teklemariam, Azadi H., Nyssen, J., Mitiku Haile, Witlox F., 2015. Commercial Farmland Acquisition in Ethiopia: The Case of Benishangul Gumuz Region. Journal of Agrarian Change, submitted.

Descheemaeker K., Nyssen J., Rossi J., Poesen J., Mitiku Haile, Moeyersons J., Deckers J., 2006. Sediment deposition and pedogenesis in exclosures in the Tigray Highlands, Ethiopia. Geoderma, 132, p. 291-314.

Descheemaeker K., Raes D., Nyssen J., Poesen J., Mitiku Haile, Deckers J., 2009. Changes in water flows and water productivity upon vegetation regeneration on degraded hillslopes in northern Ethiopia: a water balance modelling exercise. The Rangeland Journal, 31, p. 237-249.

Dessalegn Rahmato, 1994. The unquiet countryside: the collapse of "socialism" and the rural agitation, 1990 and 1991. In Abebe Zegeye and S. Pausewang (Eds), Ethiopia in change. Peasantry, Nationalism and Democracy. British Academic Press, London.

Eshetu Z., Högberg P., 2000. Reconstruction of forest site history in Ethiopian highlands based on ${ }^{13} \mathrm{C}$ natural abundance of soils. Ambio, 29, p. 83-89.

Frankl A., Jacob M., Mitiku Haile, Poesen J., Deckers J., Nyssen J., 2013. The effect of rainfall on the spatio-temporal variability of cropping systems and duration of the crop cover in the Northern Ethiopian Highlands. Soil Use and Management, 29, p. 374-383.

Frankl A., Nyssen J., De Dapper M., Mitiku Haile, Billi P., Munro R.N., Deckers J., Poesen J., 2011. Linking long-term gully and river channel dynamics to environmental change using repeat photography (North Ethiopia). Geomorphology, 129, p. 238-251.

Frankl A., Poesen J., Deckers J., Mitiku Haile, Nyssen J., 2012. Gully head retreat rates in the semiarid highlands of Northern Ethiopia. Geomorphology, 173-174, p. 185-195.

Frankl A., Seghers V., Stal C., De Maeyer P., Petrie G., Nyssen J., 2015. Using image-based 3D modelling to produce a 1935 ortho-mosaic of the Ethiopian Highlands. International Journal of the Digital Earth: online early view.

Funk C., Rowland J., Eilerts G., Emebet Kebebe, Nigist Biru, White L., Gideon Galu, 2012. A climate trend analysis of Ethiopia. US Geological Survey, Fact Sheet, 3053. 
Gascon A., 1998. La forêt perdue d'Éthiopie, un mythe contemporain. In Chastanet M., (éd.), Plantes et paysages d'Afrique, une histoire à explorer. Karthala, Paris, France. p. 383-409.

Gebeyehu Taye, Poesen J., Van Wesemael B., Vanmaercke M., Daniel Teka, Deckers J., Goosse T., Maetens W., Nyssen J., Hallet V., Nigussie Haregeweyn, 2013. Effects of land use, slope gradient; and soil and water conservation techniques, on runoff and soil loss in semi-arid northern Ethiopia. Physical Geography, 34, p. 236-259.

Gebremedhin Berhane, Martens K., Al Farrah N., Walraevens K., 2013. Water leakage investigation of micro-dam reservoirs in Mesozoic sedimentary sequences in Northern Ethiopia. Journal of African Earth Sciences, 79, p. 98-110.

Gobeze T., Bekele M., Mulugeta Lemenih, Kassa H., 2009. Participatory forest management and its impacts on livelihoods and forest status: the case of Bonga forest in Ethiopia. International Forestry Review, 11, p. 346-358.

Heltberg R., Siegel P.B., Jorgensen S.L., 2009. Addressing human vulnerability to climate change: toward a 'no-regrets' approach. Global Environmental Change, 19, p. 89-99.

Herweg K., Ludi E., 1999. The performance of selected soil and water conservation measures--case studies from Ethiopia and Eritrea. Catena, 36, p. 99-114.

Herweg K., Stillhardt B., 1999. The variability of soil erosion in the Highands of Ethiopia and Eritrea. Soil Conservation Research Project, Research report, 42. Centre for Development and Environment, University of Berne, Berne.

Holmberg J., 1977. Grain marketing and land reform in Ethiopia: an analysis of the marketing and pricing of food grains in 1976 after the land reform. Research report. Nordiska Afrikainstitutet, Uppsala.

Hurni H., 1975. Bodenerosion in Semien-Aethiopien (mit Kartenbeilage 1:25 000). Geographica Helvetica, 4, p. 157-168.

Hurni H., 1978. Soil erosion forms in the Simen mountains - Ethiopia (with map 1:25 000). Geographica Bernensia, G8, p. 93-100.

Hurni H., 1983. Soil erosion and soil formation in agricultural ecosystems Ethiopia and Northern Thailand. Mountain research and development, 3, p. 131-142.

Jacob M., Frankl A., Beeckman H., Gebrekidan Mesfin, Hendrickx M., Etafa Guyassa, Nyssen J., 2015. North Ethiopian afro-alpine treeline dynamics and forest cover change since the early 20th century. Land Degrad. Develop.: online early view.

Koehn P., 1979. Ethiopia: famine, food production, and changes in the legal order. African Studies Review, 22, p. 51-71.

Kraaijvanger R., Veldkamp T., 2015. Grain productivity, fertilizer response and nutrient balance of farming systems in Tigray, Ethiopia: a multi-perspective view in relation to soil fertility degradation. Land Degrad. Develop.: online early view.

Kumasi T., Asenso-Okyere K., 2011. Responding to Land Degradation in the Highlands of Tigray, Northern Ethiopia. IFPRI Discussion Paper, 01142. International Food Policy Research Institute.

Lamb H.F, Leng M.J, Telford R.J, Ayenew T., Umer M., 2007. Oxygen and carbon isotope composition of authigenic carbonate from an Ethiopian lake: a climate record of the last 2000 years. The Holocene, 17, p. 517-526.

Lanckriet S., Derudder B., Naudts J., Bauer H., Deckers J., Mitiku Haile, Nyssen J., 2015. A political ecology perspective of land degradation in the north Ethiopian highlands. Land Degradation \& Development: online early view. 
Lanckriet S., Tesfay Araya, Cornelis W., Verfaillie E., Poesen J., Govaerts B., Bauer H., Deckers S., Mitiku Haile, Nyssen J., 2012. Impact of conservation agriculture on catchment runoff and soil loss under changing climate conditions in May Zeg-zeg (Ethiopia). Journal of Hydrology, 475, p. 336-349.

Lavers T., 2012. Patterns of agrarian transformation in Ethiopia: State mediated commercialization and the 'land grab'. Journal of Peasant Studies, 39, p. 795-822.

Logan W., 1946. An introduction to the forests of Central and Southern Ethiopia. Institute Paper 24. Imperial Forestry Institute, University of Oxford, Oxford, UK.

McCann J., 1998. A tale of two forests: narratives of deforestation in Ethiopia, 1840-1996. African Studies Center Working Paper 209. Boston University, Boston, Massachusetts, USA.

McCartney M.P., Menker M., 2012. Evaluating the downstream implications of planned water resource development in the Ethiopian portion of the Blue Nile River. Water International, 37, p. 362-379.

McHugh O.V., Steenhuis T.S., Berihun Abebe, Fernandes E., 2007. Performance of in situ rainwater conservation tillage techniques on dry spell mitigation and erosion control in the drought-prone North Wello zone of the Ethiopian highlands. Soil and Tillage Research, 97, p. 19-36.

Meheretu Yonas, Sluydts V., Kiros Welegerima, Bauer H., Mekonen Teferi, Gidey Yirga, Mulungu L., Mitiku Haile, Nyssen J., Deckers J., Makundi R., Leirs H., 2014. Rodent abundance, stone bund density and its effects on crop damage in the Tigray highlands, Ethiopia. Crop Protection, 55, p. 61-67.

Merla G., Minucci E., 1938. Missione geologica nel Tigrai. Reale Accademia d'Italia, Roma.

Mitiku Haile, Herweg K., Stillhardt B., 2006. Sustainable Land Management - a new Approach to Soil and Water Conservation in Ethiopia. Mekelle University, Mekelle, Ethiopia; University of Berne, Berne, Switzerland.

MoA., 2012. Agricultural Investment Land Rent Directive [in Amharic]. Ministry of Agriculture: Addis Ababa, Ethiopia.

Moeyersons J., Van Den Eeckhaut M., Nyssen J., Tesfamichael Gebreyohannes, Van de Wauw J., Hofmeister J., Poesen J., Deckers J., Mitiku Haile, 2008. Mass movement mapping for geomorphological understanding and sustainable development: Tigray, Ethiopia. Catena, 75, p. 45-54. http://dx.doi.org/10.1016/j.catena.2008.04.004

Moise A., Hudson D., 2008. Probabilistic predictions of climate change for Australia and southern Africa using the reliability ensemble average of IPCC CMIP3 model simulations. Journal of Geophysical Research: Atmospheres (1984-2012), 113, D15.

Monsieurs E., Dessie M., Adgo E., Poesen J., Deckers J., Verhoest N., Nyssen J., 2015. Seasonal surface drainage of sloping farmland: a review of its hydrogeomorphic impacts. Land Degradation \& Development, 26(1), p. 35-44.

Niang I., Ruppel O.C., Abdrabo M.A., Essel A., Lennard C., Padgham J., Urquhart P., 2014. Africa. In V.R. Barros et al. (Eds), Climate Change 2014: Impacts, Adaptation, and Vulnerability. Part B: Regional Aspects. Contribution of Working Group II to the Fifth Assessment Report of the Intergovernmental Panel of Climate Change. Cambridge University Press, Cambridge, United Kingdom and New York, NY, USA, p. 1199-1265.

Nigussie Haregeweyn, Poesen J., Nyssen J., Govers G., Verstraeten G., de Vente J., Deckers J., Moeyersons J., Haile M. 2008. Sediment yield variability in Northern Ethiopia: A quantitative 
analysis of its controlling factors. Catena, 75, p. 65-76. http://dx.doi.org/10.1016/j.catena. 2008.04.011

Nigussie Haregeweyn, Poesen J., Verstraeten G., Govers G., de Vente J., Nyssen J., Deckers J., Moeyersons J., 2013. Assessing the performance of a spatially distributed soil erosion and sediment delivery model (WATEM/SEDEM) in Northern Ethiopia. Land Degradation \& Development, 24, p. 188-204.

Nyssen J., Clymans W., Descheemaeker K., Poesen J., Vandecasteele I., Vanmaercke M., Mitiku Haile, Nigussie Haregeweyn, Moeyersons J., Martens K., Amanuel Zenebe, Van Camp M., Tesfamichael Gebreyohannes, Deckers J., Walraevens K., 2010. Impact of soil and water conservation on catchment hydrological response - a case in northern Ethiopia. Hydrological Processes, 24, p. 1880-1895.

Nyssen J., Clymans W., Poesen J., Vandecasteele I., De Baets S., Nigussie Haregeweyn, Naudts J., Amanuel Hadera, Moeyersons J, Mitiku Haile, Deckers J., 2009a. How soil conservation affects the catchment sediment budget - a comprehensive study in the north Ethiopian highlands. Earth Surface Processes and Landforms, 34, p. 1216-1233.

Nyssen J., Frankl A., Mitiku Haile, Hurni H., Descheemaeker K., Crummey D., Ritler A., Portner B., Nievergelt B., Moeyersons J., Munro R.N., Deckers J., Billi P., Poesen J., 2014. Environmental conditions and human drivers for changes to north Ethiopian mountain landscapes over 145 years. Science of the Total Environment, 485-486, p. 164-179.

Nyssen J., Mitiku Haile, Nauds J., Munro N., Poesen J., Moeyersons J., Frankl A., Deckers J., Pankhurst R., 2009b. Desertification? Northern Ethiopia re-photographed after 140 years. Science of the Total Environment, 407, p. 2749-2755.

Nyssen J., Naudts J., De Geyndt K., Mitiku Haile, Poesen J., Moeyersons J., Deckers J., 2008a. Soils and land use in the Tigray highlands (Northern Ethiopia). Land Degradation \& Development, 19, p. 257-274.

Nyssen J., Poesen J., Descheemaeker K., Nigussie Haregeweyn, Mitiku Haile, Moeyersons J., Frankl A., Govers G., Munro R.N., Deckers J., 2008b. Effects of region-wide soil and water conservation in semi-arid areas: the case of northern Ethiopia. Zeitschrift für Geomorphologie, 52, p. 291-315.

Nyssen J., Poesen J., Desta Gebremichael, Vancampenhout K., D'Aes M., Gebremedhin Yihdego, Govers G., Leirs H., Moeyersons J., Naudts J., Nigussie Haregeweyn, Mitiku Haile, Deckers J., 2007. Interdisciplinary on-site evaluation of stone bunds to control soil erosion on cropland in Northern Ethiopia. Soil and Tillage Research, 94, p. 151-163.

Nyssen J., Poesen J., Mitiku Haile, Moeyersons J., Deckers J., Hurni H., 2009c. Effects of land use and land cover on sheet and rill erosion rates in the Tigray Highlands, Ethiopia. Zeitschrift für Geomorphologie, 53, p. 171-197.

Nyssen J., Poesen J., Moeyersons J., Deckers J., Haile M., Lang A., 2004. Human impact on the environment in the Ethiopian and Eritrean highlands - a state of the art. Earth-Science Reviews, 64, p. 273-320.

Nyssen J., Poesen J., Moeyersons J., Lavrysen E., Mitiku Haile, Deckers J., 2002. Spatial distribution of rock fragments in cultivated soils in northern Ethiopia as affected by lateral and vertical displacement processes. Geomorphology, 43, p. 1-16.

Nyssen J., Poesen J., Veyret-Picot M., Moeyersons J., Mitiku Haile, Deckers J., Dewit J., Naudts J., Kassa Teka, Govers G., 2006. Assessment of gully erosion rates through interviews and measurements: a case study from Northern Ethiopia. Earth Surf. Proc. Landf., 31, p. 167-185. 
Pankhurst R., 1995. The history of deforestation and afforestation in Ethiopia prior to World War I. Northeast African Studies, 2, p. 119-133.

Pietsch D., Machado M.J., 2014. Colluvial deposits - proxies for climate change and cultural chronology. A case study from Tigray, Ethiopia. Zeitschrift für Geomorphologie, Supplementary Issues, 58, p. 119-136.

Ritler A., 2003. Forests, land use and landscape in the Central and Northern Ethiopian Highlands, 1865 to 1930. Geographica Bernensia, African Studies Series A19, University of Bern, Bern, Switzerland.

SCRP., 2000. Long-term Monitoring of the Agricultural Environment in Six Research Stations in Ethiopia. Soil Erosion and Conservation Database. 7 Volumes. Soil Conservation Research Programme, Berne and Addis Ababa.

Selamyihun Kidanu, Tekalign Mamo, Stroosnijder L., 2005. Biomass production of Eucalyptus boundary plantations and their effect on crop productivity on Ethiopian highland Vertisols. Agroforestry Systems, 63, p. 281-290.

Shongwe M.E., van Oldenborgh G.J., van den Hurk B., van Aalst M., 2011. Projected changes in mean and extreme precipitation in Africa under global warming. Part II: East Africa. Journal of Climate, 24, p. 3718-3733.

Tazebe Beyene, Lettenmaier D.P., Kabat P., 2010. Hydrologic impacts of climate change on the Nile River Basin: implications of the 2007 IPCC scenarios. Climatic Change, 100, p. 433-461.

Tebebu T.Y., Abiy A.Z., Dahlke H.E., Easton Z.M., Zegeye A.D., Tilahun S.A., Collick A.S., Kidnau S., Moges S., Dadgari F., Steenhuis T.S., 2010. Surface and subsurface flow effect on permanent gully formation and upland erosion near Lake Tana in the Northern Highlands of Ethiopia. Hydrol. Earth Syst. Sci. Discuss., 7, p. 5235-5265.

Tesfaalem Gebreyohannes, Frankl A., Mitiku Haile, Nyssen J., 2015. Catchment rehabilitation and hydro-geomorphic characteristics of mountain streams in the western Rift Valley escarpment of Northern Ethiopia. Land Degradation and Development: online early view.

Tesfay Araya, Cornelis W.M., Nyssen J., Govaerts B., Fekadu Getnet, Bauer H., Kassa Amare, Raes D., Mitiku Haile, Deckers J., 2012. Medium-term effects of Conservation Agriculture based cropping systems for sustainable soil and water management and crop productivity in the Ethiopian highlands. Field Crops Res., 132, p. 53-62

Tilashwork Chanie, Collick A.S., Enyew Adgo, Lehmann C.J., Steenhuis T.S., 2013. Ecohydrological impacts of Eucalyptus in the semi humid Ethiopian Highlands: the Lake Tana Plain. Journal of Hydrology and Hydromechanics, p. 21-29.

Van Den Eeckhaut M., Moeyersons J., Nyssen J., Amanuel Zenebe, Poesen J., Mitiku Haile, Deckers J., 2009. Spatial patterns of old, deep-seated landslides: A case-study in the northern Ethiopian highlands. Geomorphology, 105, p. 239-252. http://dx.doi.org/10.1016/j.geomorph.2008.09.027

Vanmaercke M., Zenebe A., Poesen J., Nyssen J., Verstraeten G., Deckers J., 2010. Sediment dynamics and the role of flash floods in sediment export from medium-sized catchments: a case study from the semi-arid tropical highlands in northern Ethiopia. Journal of Soils and Sediments, 10, p. 611-627.

Virgo K.J., Munro R.N., 1978. Soil and erosion features of the Central Plateau region of Tigrai, Ethiopia. Geoderma, 20, p. 131-157.

Von Breitenbach F., 1961. Forests and woodlands of Ethiopia, a geobotanical contribution to the knowledge of the principal plant communities of Ethiopia, with special regard to forestry. Ethiopian Forest Review, 1, p. 5-16. 
Walraevens K., Vandecasteele I., Martens K., Nyssen J., Moeyersons J., Tesfamichael Gebreyohannes, De Smedt F., Poesen J., Deckers J., Van Camp M., 2009. Groundwater recharge and flow in a small mountain catchment in Northern Ethiopia. Hydrological Sciences Journal, 54, p. 739-753.

Wildemeersch J., Garba M., Sabiou M., Cornelis W., 2015. Water and soil conservation for improved crop productivity, water household and soil quality in Niger. Land Degrad. Develop., in press.

Wøien H., 1995. Deforestation, information and citations: a comment on environmental degradation in Highland Ethiopia. Geojournal, 37, p. 501-512.

Wood AP. 1993. Natural resource conflicts in south-west Ethiopia: state, communities, and the role of the National Conservation Strategy in the search for sustainable development. Nordic Journal of African Studies, 2, p. 83-99.

Zenebe Mekonnen, Habtemariam Kassa, Mulugeta Lemenh, Campbell B., 2007. The role and management of eucalyptus in Lode Hetosa district, Central Ethiopia. Forests, Trees and Livelihoods, 17, p. 309-323.

Zerihun Berhane, Prowse M., 2013. Climate-Change Adaptation in Ethiopia: To What Extent Does Social Protection Influence Livelihood Diversification? Development Policy Review, 31, p. o35-056.

\section{RÉSUMÉS}

Cet article de synthèse s'intéresse au passé et au futur de la gestion des terres en Éthiopie, mettant l'accent sur la partie Nord du pays. Comment l'état des sols et des forêts s'est-il modifié au cours du siècle dernier? Comment ces modifications affectent-elles les moyens de subsistance de la population? A la fin du XIX ${ }^{\mathrm{e}}$ siècle, il existait déjà des ravines de ruissellement au Nord de l'Éthiopie, bien que celles-ci se soient stabilisées vers 1935. Dans les années 1960, une recrudescence de ravinements s'est manifestée. Depuis environ 2000, les vitesses d'érosion décroissent de nouveau, en liaison avec une augmentation des activités de conservation et une couverture végétale améliorée. Par conséquent, la production totale de céréales en Éthiopie est désormais plus élevée que jamais, et la production par habitant entre 2005 et 2010 s'est élevée de $60 \%$ par rapport à celle observée entre 1985 et 1990. On assiste actuellement à l'augmentation des exportations de fleurs et de légumes, ainsi qu'à l'essor de l'exportation d'un narcotique doux, le khat, qui nécessite de grandes quantités d'eau. Les transactions foncières internationales, qui par ailleurs stagnent bien en deçà des objectifs initiaux, n'ont pas apporté d'amélioration aux moyens de subsistance des populations locales.

This article considers past and future of land management in Ethiopia, focusing on the northern part of the country. How did soils and forests change over the last century? How do these changes affect the means of subsistence of the population? At the end of the $19^{\text {th }}$ century, there were already gullies in northern Ethiopia, although these had been stabilized around 1935. In the 1960 s a reactivation of gullies has occurred. Since about 2000, erosion rates decrease again, in conjunction with an increase in conservation activities and improved vegetation cover. As a result, the total production of cereals in Ethiopia is now higher than ever, and production per capita for $2005-2010$ rose by $60 \%$ as compared to $1985-1990$. Currently there is an increase of exportations of flowers and vegetables, as well as a strong increase in the export of the mild narcotic khat, which requires large amounts of water for growing. International land transactions, which stagnate well below the initial objectives, have not brought improvement to the livelihood of local populations. 


\section{INDEX}

Mots-clés : érosion des sols, transactions foncières internationales, production céréalière, dégradation des terres

Keywords : soil erosion, international land transactions, cereal production, land degradation

\section{AUTEURS}

\section{JAN NYSSEN}

Jan Nyssen, jan.nyssen@ugent.be, est Professeur au Département de Géographie de l'Université de Gand, Belgique. Il a publié récemment :

- Jacob M., Frankl, A., Beeckman H., Gebrekidan Mesfin, Hendrickx M., Etefa Guyassa, Nyssen J., 2015. North Ethiopian afro-alpine treeline dynamics and forest cover change since the early 20th century. Land Degrad. Develop., online early view.

- Lanckriet S., Derudder B., Naudts J., Bauer H., Deckers J., Mitiku Haile, Nyssen J., 2015. A political ecology perspective of land degradation in the North Ethiopian Highlands. Land Degradation and Development, online early view.

- Nyssen J., Frankl A., Mitiku Haile, Hurni H., Descheemaeker K., Crummey D., Ritler A., Portner B., Nievergelt B., Moeyersons, J., Munro R.N., Deckers J., Billi P., Poesen J., 2014. Environmental conditions and human drivers for changes to north Ethiopian mountain landscapes over 145 years. Science of the Total Environment, 485-486 (164-179).

\section{AMAURY FRANKL}

Amaury Frankl, amaury.frankl@ugent.be, est Docteur-Assistant au Département de Géographie, Université de Gand, Belgique. Il a publié récemment :

- Frankl A., Deckers J., Moulaert L., Van Damme A., Mitiku Haile, Poesen J., Nyssen J., 2015. Integrated solutions for combating gully erosion in areas prone to soil piping: innovations from the drylands of Northern Ethiopia. Land Degradation \& Development, online early view.

- Frankl A., Seghers V, Stal C., De Maeyer Ph., Petrie G., Nyssen J., 2015. Using image-based modelling (SfM-MVS) to produce a 1935 ortho-mosaic of the Ethiopian Highlands. International Journal of the Digital Earth, online early view.

- Frankl A., Nyssen J., De Dapper M., Mitiku Haile, Billi P., Munro R.N., Deckers J., Poesen J., 2011. Linking long-term gully and river channel dynamics to environmental change using repeat photography (North Ethiopia). Geomorphology, 129 (3-4), p. 238-251.

\section{JEAN POESEN}

Jean Poesen, jean.poesen@ees.kuleuven.be, est Professeur au Département des Sciences de la Terre et de l'Environnement, KU Leuven, Belgique. Il a publié récemment :

- Monsieurs E., Poesen J., Mekete Dessie, Enyew Adgo, Verhoest N., Deckers J., Nyssen J., 2015. Effects of drainage ditches and stone bunds on topographical thresholds for gully head development in North Ethiopia. Geomorphology, 234, p. 193-203.

- Vanmaercke M., Poesen J., Broeckx J., Nyssen J., 2014. Sediment Yield in Africa. Earth Science Reviews, 136, p. 350-368.

- Getahun K., Van Rompaey A., Van Turnhout P., Poesen J., 2013. Factors controlling patterns of deforestation in moist evergreen Afromontane forests of Southwest Ethiopia. Forest Ecology and Management, 304, p. 171-181. 


\section{AMANUEL ZENEBE}

Amanuel Zenebe, amanuelzenebe@gmail.com, est Chargé de Cours au Département de la Protection des Ressources Terrestres et de l'Environnement à l'Université de Mekelle, Ethiopie· Il a publié récemment :

- Amanuel Zenebe, Vanmaercke M., Poesen J., Verstraeten G., Nigussie Haregeweyn, Mitiku Haile, Kassa Amare, Deckers J., Nyssen J., 2013. Spatial and temporal variability of river flows in the degraded semi-arid tropical mountains of northern Ethiopia. Zeitschrift für Geomorphologie, 57(2), p. $143-169$.

- Habtamu Temesgen, Nyssen, J., Amanuel Zenebe, Nigussie Haregeweyn, Mengistie Kindu, Mulugeta Lemenih, Mitiku Haile, 2013. Ecological succession and land use changes in a lake retreat area (Main Ethiopian Rift Valley). Journal of Arid Environments, 91, p. 53-60.

- Vanmaercke, M., Amanuel Zenebe, Poesen, J., Nyssen, J., Verstraeten, G., Deckers, J., 2010. Sediment dynamics and the role of flash floods in sediment export from medium-sized catchments: a case study from the semi-arid tropical highlands in northern Ethiopia. Journal of Soils and Sediments, 10(4), p. 611-627.

\section{JOZEF DECKERS}

Jozef Deckers, seppe.deckers@ees.kuleuven.be, est Professeur au Département des Sciences de la Terre et de l'Environnement, KU Leuven, Belgique. Il a publié récemment :

- Mekonen Teferi, Declerck S., De Bie T., Lemmens P., Abraha Gebrekidan, Tsehaye Asmelash, Tadesse Dejenie, Kindeya Gebrehiwot, BauerH., Deckers J., Snoeks J., De Meester L., 2014. Strong effects of occasional drying on subsequent water clarity and cyanobacterial blooms in cool tropical reservoirs. Freshwater Biology, 59, p. 870-877.

- Lenaerts L., Breusers M., Dondeyne S., Bauer H., Mitiku Haile, Deckers J., 2014. 'This pasture is ours since ancient times': An ethnographic analysis of the reduction in conflicts along the post-1991 Afar-Tigray regional boundary. Journal of Modern African Studies, 52, p. 25-44.

- Meheretu Yonas, Sluydts V., Kiros Welegerima, Bauer H., Mekonen Teferi, Gidey Yirga, Mulungu L., Mitiku Haile, Nyssen J., Deckers J., Makundi R., Leirs H., 2014. Rodent abundance, stone bund density and its effects on crop damage in the Tigray highlands, Ethiopia. Crop Protection, 55, p. 61-67. 